\title{
Promover la regulación individual y grupal del aprendizaje en entornos colaborativos: una experiencia en Educación Superior
}

Javier Onrubia $^{1}$, María José Rochera ${ }^{1}$, Anna Engel ${ }^{1}$

${ }^{1}$ Dpt. de Psicología Evolutiva y de la Educación

Universitat de Barcelona, Barcelona

\section{España}

Correspondencia: Javier Onrubia. P. Vall d'Hebron, 17108035 Barcelona. España. E-mail: javier.onrubia@ub.edu

(C) Education \& Psychology I+D+i and Ilustre Colegio Oficial de la Psicología de Andalucía Oriental (Spain) 


\section{Resumen}

En este trabajo presentamos una experiencia de innovación docente dirigida a promover la regulación individual y grupal del aprendizaje en estudiantes universitarios que trabajan en un entorno colaborativo mediado por ordenador. Participaron en la experiencia 127 estudiantes y tres profesores de una asignatura troncal de Psicología de la Educación de la Universitat de Barcelona (España). La experiencia se basó en el uso de una herramienta digital el "cuaderno de bitácora"- en que los estudiantes debían incluir una serie de evidencias de su proceso de trabajo personal y grupal, y del progreso de su aprendizaje, que eran revisadas y comentadas sistemáticamente por los profesores. Se valoró la experiencia a partir de cuestionarios a los estudiantes y autoinformes de los profesores, así como de las calificaciones de los estudiantes. Los resultados de la experiencia fueron positivos tanto desde el punto de vista de la mejora en la organización y funcionamiento individual y grupal de los estudiantes como de la satisfacción de los estudiantes y el profesorado.

Palabras Clave: aprendizaje autorregulado, enseñanza superior, entorno colaborativo, innovación pedagógica. 


\title{
Promoting individual and group regulated learning in colaborative settings: an experience in higher education
}

\begin{abstract}
We present a teaching innovation intervention aimed at promoting individual and group learning regulation in undergraduate students working on a computer supported collaborative learning environment. Participants were 127 students and three teachers of a compulsory course on Educational Psychology at the University of Barcelona (Spain). As a central point of the intervention, a digital tool ---the "student's log"--- was designed for students to include a series of evidences of their individual and group work processes, and of their learning progress, which were systematically revised and commented by the teachers. The intervention was evaluated using questionnaires to the students and teachers' self-reports, and also considering students' final grades. The results of the intervention were positive both from the point of view of students' learning and working processes and from the point of view of students' and teachers' satisfaction.
\end{abstract}

Keywords: self-regulated learning, higher education, educational innovation, collaborative environment

Reception: 06/04/14 Initial acceptance: 08/02/14 Final acceptance: 03/14/15 


\section{Introducción}

A lo largo de las últimas décadas la investigación psicoeducativa ha demostrado la potencialidad de la colaboración entre estudiantes para mejorar los procesos de construcción del conocimiento y de atribución de sentido a los contenidos y actividades de enseñanza y aprendizaje (Dillenbourg, Baker, Blaye y O'Malley, 1996; Johnson y Johnson, 1974; Slavin, 1996). Igualmente, ha demostrado que dicha potencialidad puede verse aumentada cuando la colaboración está mediada por tecnologías digitales (Dillenbourg, Järvelä y Fischer, 2009; Stahl, Koschmann y Suthers, 2006; Resta y Laferrière, 2007). El interés por analizar esta potencialidad ha llevado al desarrollo de un nuevo ámbito de estudio que se ha dado en denominar aprendizaje colaborativo mediado por ordenador (Computer-Supported Colaborative Learning, CSCL).

Colaborar para aprender permite a los estudiantes involucrarse en formas de conversación que pueden llevarles a desarrollar niveles cada vez más altos de intersubjetividad y, con ello, a ampliar y enriquecer de manera progresiva los sistemas de significados que construyen conjuntamente sobre las tareas y los contenidos de enseñanza y aprendizaje (Bereiter, 1994; Mercer, 2001; Scardamalia y Bereiter, 2003; Suthers, 2006). Igualmente, permite que los estudiantes negocien y se pongan de acuerdo sobre la organización y evolución de la propia actividad conjunta, planificando, coordinando y supervisando mutuamente el trabajo común, así como sus aportaciones y roles en la interacción (Engel y Onrubia, 2010; Manlove, Lazonder y de Jong, 2009; Meier, Spada y Rummel, 2007).

Sin embargo, concretar estas potencialidades no es fácil. La investigación también ha constatado de forma reiterada, en particular en el caso de los entornos CSCL (Dillenbourg, Järvelä y Fischer, 2009; Stahl, Koschmann y Suthers, 2006; Resta y Laferrière, 2007), que no basta con poner a los estudiantes a trabajar en grupo ni con ofrecerles potentes "tecnologías colaborativas" (Lipponen y Lallimo, 2004) para que emerjan los mecanismos y las pautas interactivas que explican la eficacia del aprendizaje colaborativo. Su aparición o no, y con ella la mayor o menor contribución de la colaboración entre estudiantes para el aprendizaje, depende en gran medida de la naturaleza de la implicación y participación de los estudiantes en el trabajo grupal. 
Por ello, la investigación en este ámbito ha ido evolucionando progresivamente hacia planteamientos que tratan de identificar maneras de apoyar instruccionalmente los procesos de colaboración entre los estudiantes. Por una parte, los investigadores se han interesado por el papel que puede jugar el profesor como guía y apoyo al proceso de construcción colaborativa del conocimiento que han de llevar a cabo los estudiantes (Dillenbourg y Hong, 2008; Garrison y Anderson, 2003; Kirschner, Sweller y Clark, 2006). Por otra, por el papel que pueden jugar las propias tecnologías digitales para apoyar y facilitar determinados aspectos de los procesos colaborativos (Lipponen y Lallimo, 2004; Martínez, Dimitriadis y de la Fuente, 2003; Pifarré y Cobos, 2010).

En ambos casos, el énfasis recae tanto en la manera de diseñar entornos o situaciones que promuevan determinados procesos interactivos entre los estudiantes como en la manera de ayudar a desarrollar los procesos tanto individuales como grupales de trabajo que desarrollan efectivamente los grupos a lo largo del proceso de enseñanza y aprendizaje. Tabak (2004) utiliza el término synergistic scaffolding para referirse a la necesidad de crear sinergias entre las ayudas y soportes que se ofrecen desde diferentes fuentes (profesor, herramientas digitales, pares, materiales de aprendizaje, etc.) dirigidas al grupo clase en su conjunto, a cada pequeño grupo o al estudiante individualmente considerado. En un sentido similar, Fischer y Dillenbourg (2006) hablan de orchestration para destacar el papel del profesor que debe diseñar por anticipado de forma armónica múltiples "voces" (actividades, apoyos y andamios) para después afinarlas y flexibilizarlas para que se adapten en todo momento a los particulares procesos colaborativos que los distintos grupos de estudiantes desarrollan (para una discusión más amplia véase Dillenbourg et al., en prensa).

Entre los distintos aspectos implicados en el trabajo colaborativo, uno de los que está recibiendo mayor atención en los últimos años desde este punto de vista es el relativo a la regulación individual y grupal del trabajo y el aprendizaje por parte de los alumnos, es decir, al control que tienen los miembros del grupo sobre sus propios procesos de colaboración y aprendizaje. Así entendida, esta regulación pasa por actividades metacognitivas de planificación, supervisión y evaluación tanto del trabajo como del aprendizaje en el grupo, y tiene un carácter tanto individual como social, incluyendo procesos de autorregulación de cada alumno, de regulación del trabajo y el aprendizaje de los otros, y de regulación conjunta o compartida entre los miembros del grupo (Jarvela y Hadwin, 2013; Volet, Summers y Thurman, 2009). Diversos trabajos recientes subrayan la complejidad de estos procesos de regulación y 
la necesidad de enseñarlos (Boeckaerts, 2002; de la Fuente y Justicia, 2007; de la Fuente, Justicia, Sander y Cardelle-Elawar, 2014; Pintrich, 2000), su importancia para el éxito del trabajo y el aprendizaje colaborativo, y las dificultades que habitualmente tienen los alumnos para regularse de manera exitosa en situaciones de trabajo colaborativo en pequeño grupo (Álvarez-Valdivia y López-Benavides, 2010; Janssen, Erkens, Kirschner y Kanselaar, 2012; Saab, Joolingen y Hout-Wolters, 2012; Schoor y Bannert, 2012).

\section{Objetivo}

En este marco, nuestro trabajo presenta una experiencia de innovación docente dirigida a promover la regulación individual y grupal del aprendizaje en estudiantes universitarios que trabajan en un entorno colaborativo mediado por ordenador. La experiencia se basa en el uso de una herramienta digital —el "cuaderno de bitácora" - diseñada para ayudar a mejorar los procesos de planificación, supervisión y revisión del trabajo y el aprendizaje individual y en grupo de los estudiantes.

\section{Metodología}

\section{Participantes}

La experiencia se llevó a cabo en una asignatura troncal del ámbito de la Psicología de la Educación impartida en los estudios de Psicología en la Universidad de Barcelona (España). Participaron dos grupos de la asignatura, con 127 estudiantes matriculados y tres profesores (uno en uno de los grupos y dos en el otro). La asignatura se organizó en tres bloques temáticos, de unas cuatro semanas de duración cada uno. El trabajo en cada bloque se estructuró en torno a la realización por parte de los estudiantes, organizados en pequeños grupos de 4-6 alumnos, de dos tareas: un caso propio de la psicología escolar y un mapa conceptual con los conceptos básicos para resolver el caso. Los grupos abordaban estas tareas de manera autónoma, aunque con un seguimiento y apoyo continuados del profesor. La colaboración entre los miembros de cada grupo se desarrollaba tanto presencial como virtualmente. El trabajo virtual se realizó a través del Campus Virtual de la Universidad de Barcelona, basado en la plataforma Moodle. Para este trabajo, cada pequeño grupo dispuso de un foro, un chat y un wiki, todos ellos accesibles sólo a los miembros del grupo y al profesor.

El abordaje autónomo de las tareas por parte de cada grupo, junto al carácter abierto y complejo de las mismas, exigía a los grupos coordinar y regular de manera continuada tanto la 
colaboración entre los diversos miembros del grupo como la aportación de cada uno de ellos al proceso de trabajo grupal. En este contexto, la experiencia se centró en el diseño y desarrollo de un conjunto de ayudas dirigidas a apoyar el proceso de regulación de los diversos grupos y a promover el desarrollo de competencias de planificación, seguimiento y evaluación del trabajo colaborativo por parte de los estudiantes. Estas ayudas se vertebraron alrededor de un instrumento al que denominamos "cuaderno de bitácora".

\section{Procedimiento e instrumentos de intervención}

El "cuaderno de bitácora" era un espacio en el aula virtual de la asignatura en el que los miembros de cada pequeño grupo debían ir incluyendo una serie de evidencias digitales de su proceso de trabajo personal y grupal, y del progreso de su aprendizaje. El término "cuaderno de bitácora" pretendía destacar su función de registro sistemático del proceso realizado y del progreso alcanzado, y al tiempo subrayar su carácter personal y propio, en términos tanto de grupo como individuales. Así, el "cuaderno..." tenía características que lo acercaban tanto a un portafolio electrónico como a un diario de aprendizaje, sin llegar sin embargo a identificarse completamente con ninguno de ellos. En el primer caso, porque se solicitaba la presentación de evidencias de distinto tipo tanto del proceso como de los resultados del propio trabajo y aprendizaje, y, en el segundo, por el carácter parcialmente público y relativamente sistemático en el tiempo de las "entradas" o aportaciones. Desde el punto de vista tecnológico, y a partir de un análisis de las posibilidades y limitaciones de las herramientas Moodle disponibles en el Campus Virtual, el "cuaderno..." se implementó sobre un foro privado de pequeño grupo, en que los miembros de cada grupo podían realizar contribuciones y adjuntar documentos.

Las evidencias solicitadas en cada uno de los bloques de la asignatura se diseñaron para favorecer la toma de conciencia por parte de los estudiantes sobre diferentes dimensiones implicadas en los procesos de regulación de la colaboración, así como para promover de manera progresiva el avance individual y grupal en el control de dichos procesos. En concreto, se consideró:

- El carácter individual o grupal de las evidencias aportadas.

- El momento del proceso de trabajo de cada una de las actividades en que debían aportarse las evidencias: al inicio del proceso (evidencias de planificación individual y grupal de las tareas a realizar); durante el proceso (evidencias relacionadas con el avance del trabajo y 
las sucesivas versiones del producto final a entregar); o al final del proceso (evidencias relacionadas con la valoración del producto final elaborado).

- El tipo de evidencias, distinguiendo entre evidencias de producto (productos parciales o versiones provisionales del producto final) y evidencias de proceso (descripciones, reflexiones, comentarios o valoraciones del proceso mismo de trabajo individual o grupal).

- El grado de reflexión sobre las propias evidencias, distinguiendo entre evidencias directas de producto o de proceso, y "meta-evidencias", es decir, comentarios o valoraciones sobre las evidencias propias o de otros compañeros, su calidad y aportaciones al proceso de trabajo individual o grupal.

Así, en el primer bloque de la asignatura se solicitó a cada grupo la inclusión en el “cuaderno..." de, al menos, cinco evidencias: dos de carácter individual (relativas a las actividades del mapa y la resolución del caso) y tres de carácter grupal (también relativas a las dos actividades). La Tabla 1 recoge, a título ilustrativo, la demanda de evidencias de este bloque tal y como se formuló a los estudiantes.

\section{Tabla 1. Evidencias solicitadas a los estudiantes para el "cuaderno de bitácora" del bloque 1 de la asignatura}

\section{CUADERNO DE BITÁCORA - BLOQUE TEMÁTICO 1}

El "cuaderno de bitácora" ha sido diseñado para que los grupos realicen anotaciones a manera de evidencias relevantes, individuales y de grupo, del estudio de los contenidos y del proceso de elaboración de las actividades del bloque temático 1 .

Estas evidencias tienen que ser aportadas por todos los miembros del grupo. En concreto, a lo largo del desarrollo de este bloque se tendrían que aportar cinco tipos de evidencia, dos de carácter individual y tres de carácter grupal, con las siguientes características:

- Cada estudiante individualmente aportará dos evidencias: una relacionada con la elaboración del mapa conceptual y la otra relacionada con la resolución del caso. Al menos una de estas dos evidencias tiene que ser un producto individual previo a la discusión de la actividad en el grupo (producto digitalizado y publicado como adjunto al mensaje que presenta la evidencia).

- El grupo aportará tres evidencias que tienen que hacer referencia a las dos actividades propuestas en el bloque temático, el mapa conceptual y el caso. Al menos dos de ellas tienen que ser versiones provisionales del producto finalmente presentado (versión provisional digitalizada y publicada como adjunto al mensaje que presenta la evidencia). Las evidencias de grupo tienen que reflejar el acuerdo y la elaboración realizada por todos los miembros del grupo.

Cada evidencia, individual o de grupo, se publicará como un mensaje en el "cuaderno de bitácora" al que, eventualmente, se adjuntará un archivo con el producto provisional solicitado. Se recomienda no incluir adjuntos en formato de imagen (gif, jpg, tif ...). Es más conveniente insertar la imagen en un documento de texto y adjuntar el archivo resultante (doc, pdf, $\mathrm{rtf}$...). Asimismo se recomienda que el asunto del mensaje haga referencia explícita al contenido de la evidencia (por ejemplo, "evidencia grupal: mapa conceptual"). 
En términos de las dimensiones antes indicadas, esta demanda ponía el énfasis en la necesidad de diferenciar evidencias individuales y grupales, pero no ponía en juego las restantes dimensiones, al no solicitar explícitamente evidencias elaboradas al inicio o al final del proceso de trabajo, evidencias de proceso o "meta-evidencias". En los dos bloques siguientes de la asignatura, la demanda se complejizó de forma progresiva, solicitando de forma explícita evidencias diversas en relación con dichas dimensiones. En el segundo bloque, en concreto, se solicitaron siete evidencias, tres de carácter individual y cuatro de carácter grupal, y se incluyó la demanda de evidencias previas y posteriores a la realización de las actividades (por ejemplo, una planificación inicial grupal del proceso de trabajo a realizar por el grupo), de evidencias de proceso (por ejemplo, un comentario sobre el proceso de trabajo del grupo en una de las actividades) y de "meta-evidencias" (por ejemplo, una valoración/reflexión, al final del proceso, sobre la utilidad de la planificación elaborada) tanto de carácter retroactivo como proactivo. En el tercer bloque, se mantuvo casi idéntica la demanda de esa diversidad de evidencias, y se aumentó su complejidad pidiendo que una de las evidencias grupales comentara y valorara lo que habían supuesto para el producto grupal las aportaciones individuales de los miembros del grupo.

El cambio de complejidad en la demanda de evidencias entre el primer bloque y los dos siguientes se apoyó con la introducción, al final del primer bloque, de una actividad presencial de valoración por parte de los pequeños grupos de la dinámica de trabajo grupal que habían tenido en el bloque. Para ello, los estudiantes respondían individualmente un pequeño cuestionario que les ayudaba a tomar conciencia y valorar diversos aspectos de ese proceso, y discutían en pequeño grupo, a partir de sus respuestas, cómo mejorarlo. Los acuerdos al respecto guiaban la planificación del trabajo del grupo que debían preparar para el segundo bloque. La elaboración de esta planificación se apoyaba también con una pauta específica. Esta actividad se repetía asimismo, de forma similar, al final del segundo bloque.

La evolución global de las evidencias solicitadas a lo largo de los tres bloques se representa gráficamente en la Figura 1. 


\begin{tabular}{|c|c|c|c|c|c|c|c|c|c|c|c|c|c|c|c|c|c|}
\hline \multirow{2}{*}{\multicolumn{2}{|c|}{ Actividad de referencia }} & \multicolumn{5}{|c|}{ BT 1} & \multicolumn{5}{|c|}{ BT 2} & \multicolumn{6}{|c|}{ BT 3} \\
\hline & & $\mathbf{M}$ & $\mathbf{C}$ & \multicolumn{3}{|c|}{ M-C } & $\mathbf{P}$ & $\mathbf{M}$ & $\mathbf{M}$ & & $\mathbf{P}$ & $\mathbf{P}$ & $\mathbf{M}$ & $\mathbf{M}$ & $\mathbf{C}$ & $\mathbf{C}$ & $\mathbf{P}$ \\
\hline \multicolumn{2}{|l|}{ Evidencia } & 1 & 2 & 3 & 4 & 5 & 1 & 2 & 3 & & 7 & 1 & 2 & 3 & 4 & 5 & 6 \\
\hline \multirow{2}{*}{ Autoría } & Individual & & & & & & & & & & & & & & & & \\
\hline & Grupal & & & & & & & & & & & & & & & & \\
\hline \multirow{3}{*}{ Momento } & Inicio & & & & & & & & & & & & & & & & \\
\hline & Durante & & & & & & & & & & & & & & & & \\
\hline & Final & & & & & & & & & & & & & & & & \\
\hline \multirow{2}{*}{ Tipo } & Producto parcial & & & & & & & & & & & & & & & & \\
\hline & Reflexión & & & & & & & & & & & & & & & & \\
\hline \multirow{2}{*}{ Grado } & evidencia directa & & & & & & & & & & & & & & & & \\
\hline & meta-evidencia & & & & & & & & & & & & & & & & \\
\hline
\end{tabular}

M: mapa conceptual; C: caso; P: planificación

Figura 1. Secuencia de evidencias solicitada en cada uno de los bloques de la asignatura

Las evidencias tanto individuales como grupales aportadas a lo largo de cada bloque por cada pequeño grupo fueron objeto de revisión, valoración y comentarios por parte del profesorado durante y al final del bloque. Las valoraciones y los comentarios se incorporaban al informe escrito de devolución que cada grupo recibía al final del bloque en relación con el conjunto de los trabajos realizados, y se discutían en tutorías presenciales destinadas a comentar dichos informes. Tanto en los informes como en las tutorías se pretendía, a partir del análisis de las evidencias aportadas por los grupos (número, tipo y calidad de las evidencias, momento en que se incorporaban al "cuaderno...", coherencia entre evidencias individuales y grupales...), favorecer la toma de conciencia de sus procesos de trabajo colaborativo y de la aportación de cada miembro a ese trabajo, así como de la relación entre estos procesos, los productos elaborados y la calificación obtenida; potenciar la reflexión sobre estos procesos; y dar orientaciones para una regulación individual y colectiva cada vez más autónoma y reflexiva del trabajo y del aprendizaje grupal.

\section{Procedimiento de recogida de datos}

La valoración de la experiencia se realizó recogiendo información tanto de los estudiantes como de los profesores participantes. En el caso de los estudiantes, la información se recogió a través de un cuestionario de respuesta individual que los estudiantes cumplimentaron al final de la asignatura. Para su construcción se tomó como punto de partida el Cuestio- 
nario de valoración y satisfacción de la asignatura, utilizado habitualmente en otras ediciones de la misma, adaptándolo a los objetivos de la innovación. El cuestionario se estructuraba en torno a tres bloques de cuestiones: la satisfacción de los estudiantes con el "cuaderno de bitácora" y las restantes actividades de apoyo a los procesos de regulación del trabajo grupal empleadas en la asignatura; su valoración de cómo había funcionado en cada uno de los bloques de la asignatura la organización de su trabajo tanto grupal como individual; y su valoración global de la asignatura. En concreto estaba formado por 22 ítems cerrados tipo Likert, con cinco valores posibles de respuesta, y siete preguntas complementarias de respuesta abierta, donde se permitía en cada caso hasta tres respuestas posibles. Como información adicional, se recogieron también las calificaciones finales obtenidas por los estudiantes.

En el caso de los profesores, elaboraron, también al final de la asignatura, un autoinforme de valoración de la experiencia. De forma similar a lo señalado para los cuestionarios, el modelo de autoinforme se elaboró tomando como base los utilizados en experiencias previas de innovación con participación de los autores, y adaptándolos a la intervención realizada en este caso. El autoinforme estaba formado por 13 preguntas: nueve ítems tipo Likert, en que se solicitaba su valoración sobre diversos aspectos de la experiencia, y cuatro preguntas de carácter abierto, en que se les pedía detallar los puntos fuertes y débiles de la experiencia, sus principales aportaciones, posibles elementos de mejora, y un comentario general final.

\section{Análisis de datos}

Para el análisis de los ítems tipo Likert de los cuestionarios de los estudiantes y los autoinformes del profesorado se utilizó estadística descriptiva. Para las preguntas de carácter abierto se realizó un análisis de contenido de las respuestas, de carácter inductivo, y se calcularon las frecuencias y porcentajes de respuesta en las diferentes categorías obtenidas.

\section{Resultados}

La valoración y satisfacción de los estudiantes con el "cuaderno..." resultó claramente positiva (Figura 2). Así, el 79\% de los estudiantes lo consideró "bastante” o "muy" útil para mejorar la organización y planificación de su trabajo individual, el 78\% para mejorar la organización y planificación de su trabajo en grupo, y también el $78 \%$ para mejorar la calidad de los productos elaborados en la asignatura. Además, el $83 \%$ de los estudiantes afirmó que el 
hecho de tener que entregar sistemática y continuadamente evidencias individuales y grupales les había ayudado "bastante" o "mucho" a regular y gestionar mejor su proceso de aprendizaje. Esta valoración positiva se extendió igualmente al resto de las actividades e instrumentos de apoyo a los procesos de regulación del trabajo grupal empleadas en la asignatura (Figura 3). A pesar de esta valoración globalmente positiva de la asignatura, los estudiantes perciben que se les exige, en conjunto, una dedicación muy elevada en tiempo y esfuerzo. Cuando se les pregunta, por ejemplo, cuántas asignaturas podrían cursar adecuadamente y de manera simultánea en un mismo semestre con el mismo nivel de carga global de trabajo y de tiempo dedicado, como media señalan entre dos y tres asignaturas - muy por debajo de las cinco o seis asignaturas por semestre que tienen asignadas típicamente en la secuencia curricular de la titulación.

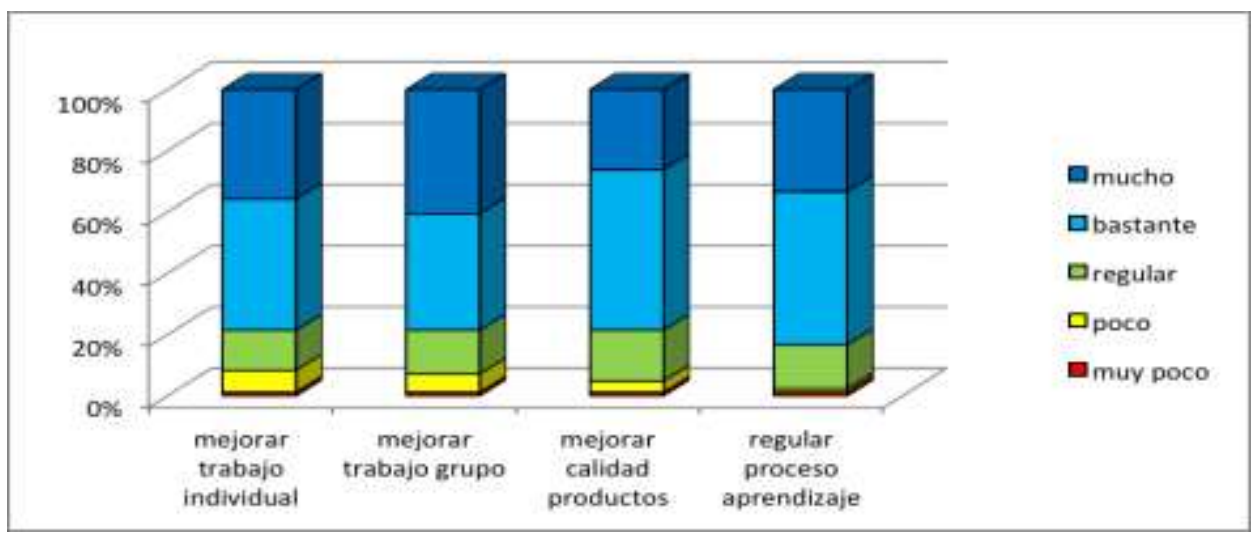

Figura 2. Valoración de los estudiantes sobre la utilidad del "cuaderno de bitácora"

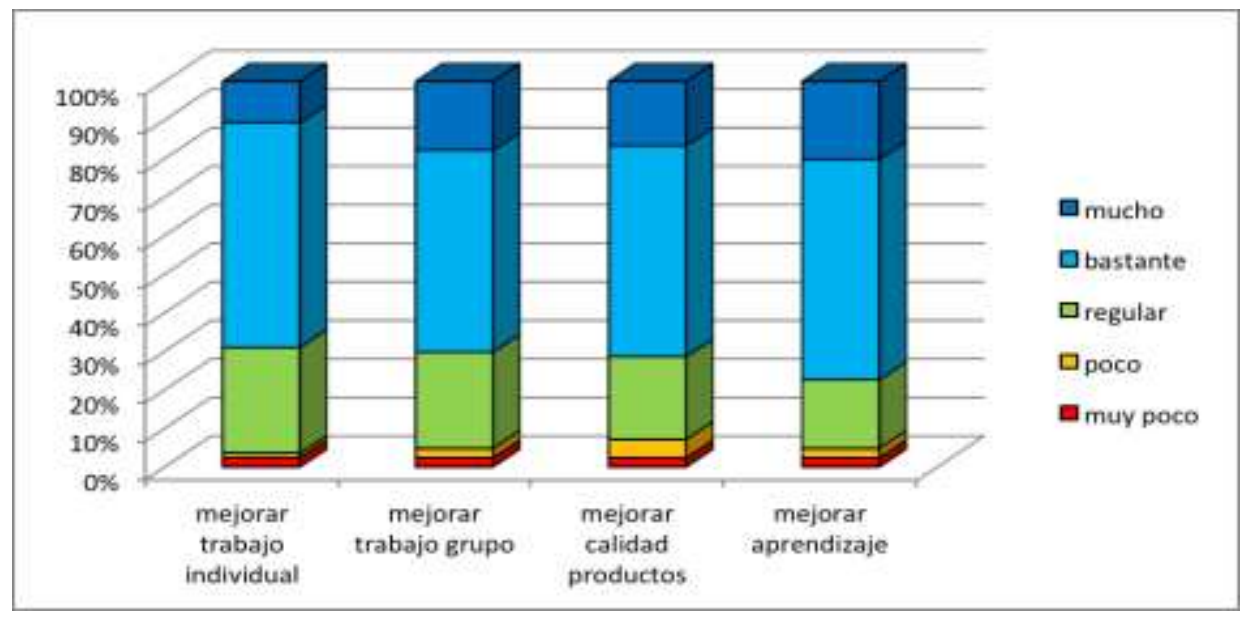

Figura 3. Valoración de los estudiantes sobre la utilidad de las actividades complementarias de apoyo a la regulación del trabajo en grupo 
Además de manifestar su satisfacción global con el "cuaderno..." y las actividades de apoyo a los procesos de regulación grupal, los estudiantes indicaron en sus respuestas a los cuestionarios que sus procesos de trabajo en grupo habían mejorado a lo largo de la asignatura (Figuras 4, 5 y 6). Así, y en cuanto a la organización y planificación del trabajo individual, sólo el 47\% de los estudiantes valoró que había sido "buena" o "muy buena" en el bloque 1 de la asignatura, mientras que ese porcentaje subió hasta el $87 \%$ en el bloque 2, y hasta el $90 \%$ en el bloque 3. En cuanto a la organización y planificación del trabajo en pequeño grupo, la consideraron "buena" o "muy buena" en el bloque 1 el 40\% de los estudiantes; en el bloque 2, el 78\%; y en el bloque 3, el 83\%. Para la articulación de las actividades individuales y en grupo, los datos fueron del $51 \%$ en el bloque 1 , el $84 \%$ en el bloque 2 y el $81 \%$ en el bloque 3 . Como se recordará, fue en los bloques 2 y 3 cuando se introdujo en el “cuaderno..." la exigencia de evidencias relacionadas con la planificación y la evaluación de los procesos de trabajo grupal, de evidencias relativas a la toma de conciencia del proceso de trabajo individual y grupal, y de "meta-evidencias". Y también fue en los bloques 2 y 3 cuando se introdujeron las actividades e instrumentos de apoyo a la revisión y planificación de la organización y funcionamiento del grupo. Los resultados apuntan, pues, un claro efecto positivo del "cuaderno...” y las restantes actividades de apoyo planteadas sobre los procesos de regulación del trabajo grupal de los estudiantes.

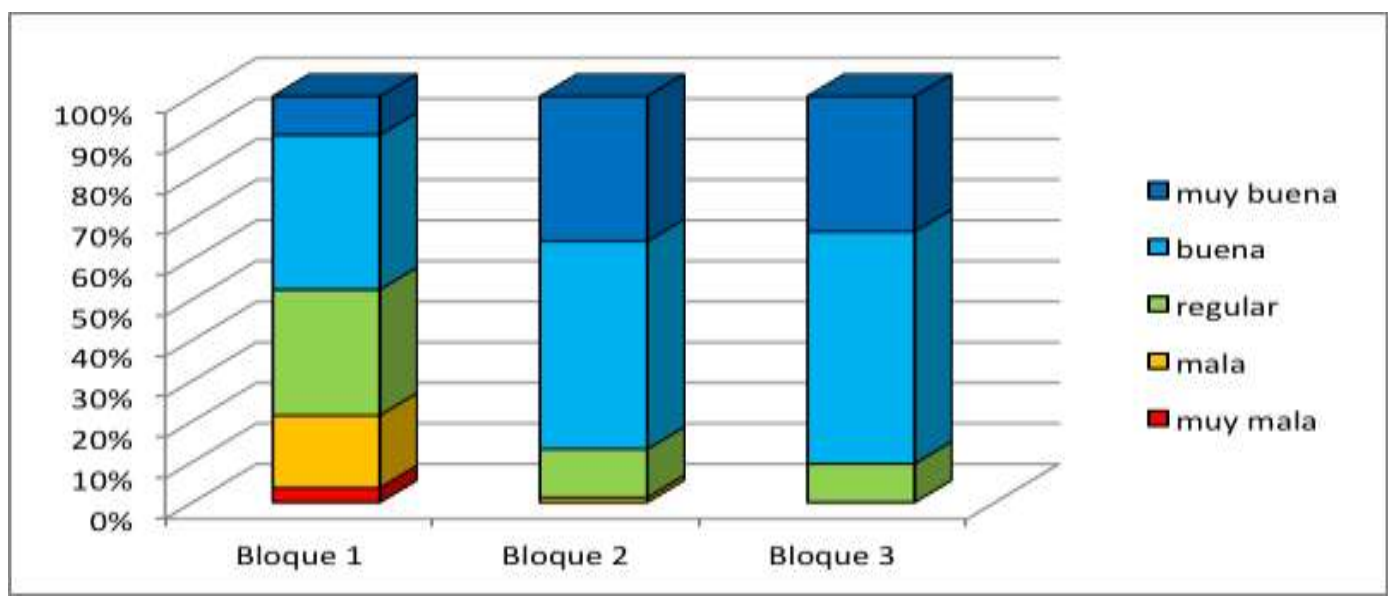

Figura 4. Valoración de los estudiantes sobre la organización y planificación de su trabajo individual en los tres bloques temáticos de la asignatura 


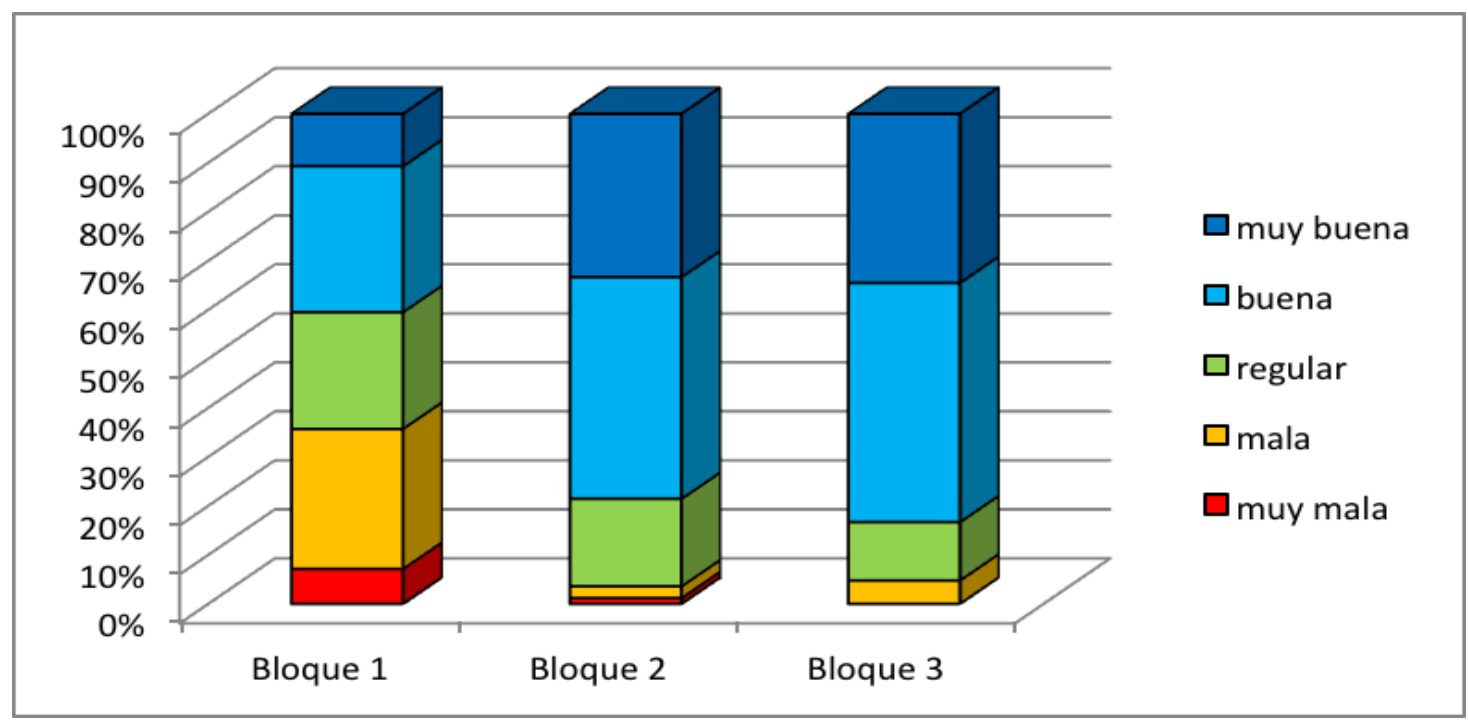

Figura 5. Valoración de los estudiantes sobre la organización y planificación de su trabajo en grupo en los tres bloques temáticos de la asignatura

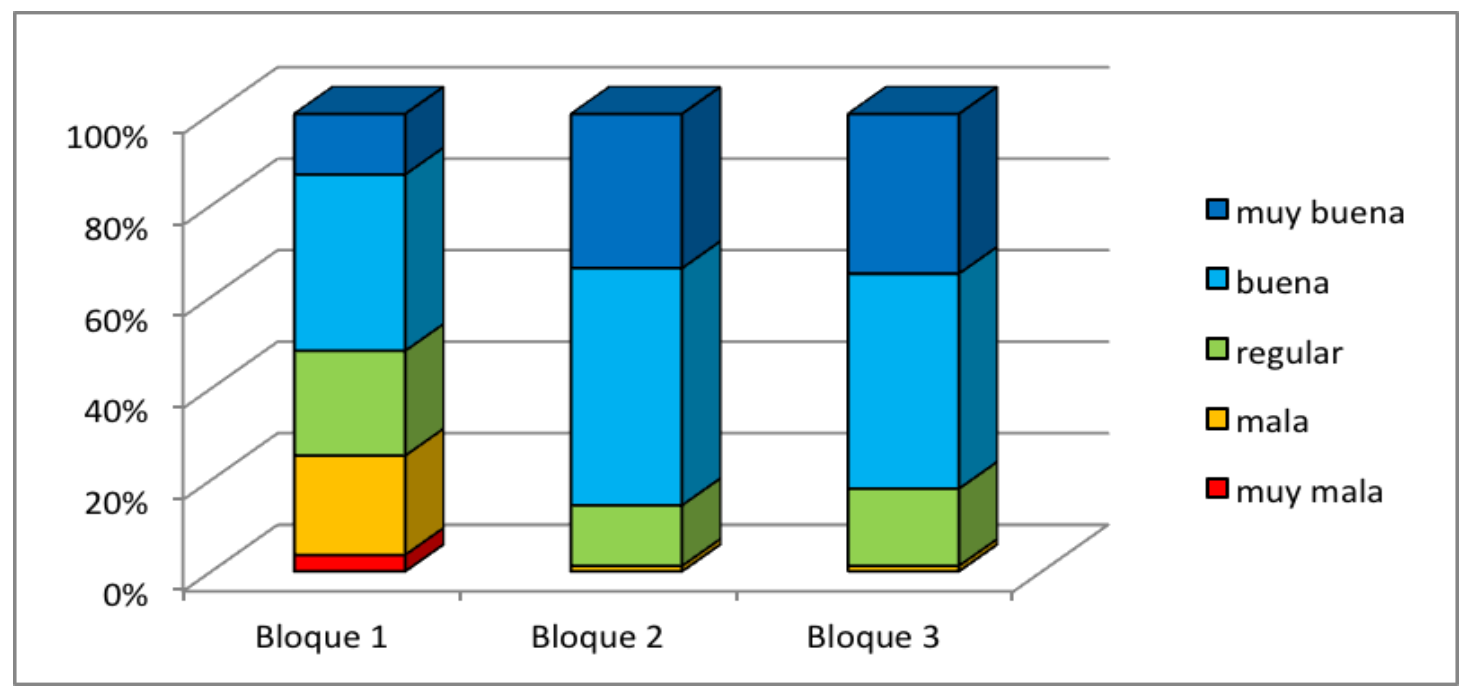

Figura 6. Valoración de los estudiantes sobre articulación de su trabajo individual y en grupo en los tres bloques temáticos de la asignatura

Los resultados anteriores se enmarcan, además, en una valoración global positiva del conjunto de la asignatura, y en unos resultados académicos finales en la misma que podemos calificar, igualmente, de positivos. Así, el $85 \%$ de los estudiantes afirmaron que la asignatura les había ayudado a aprender de manera significativa "más" o "mucho más" que asignaturas con un planteamiento metodológico más tradicional, y el $87 \%$ de los estudiantes consideraron la experiencia "muy satisfactoria" o "bastante satisfactoria" (Figuras 7 y 8). Igualmente, el $80 \%$ respondieron afirmativamente a la pregunta de si volverían a matricularse en una asignatura con un planteamiento similar. 


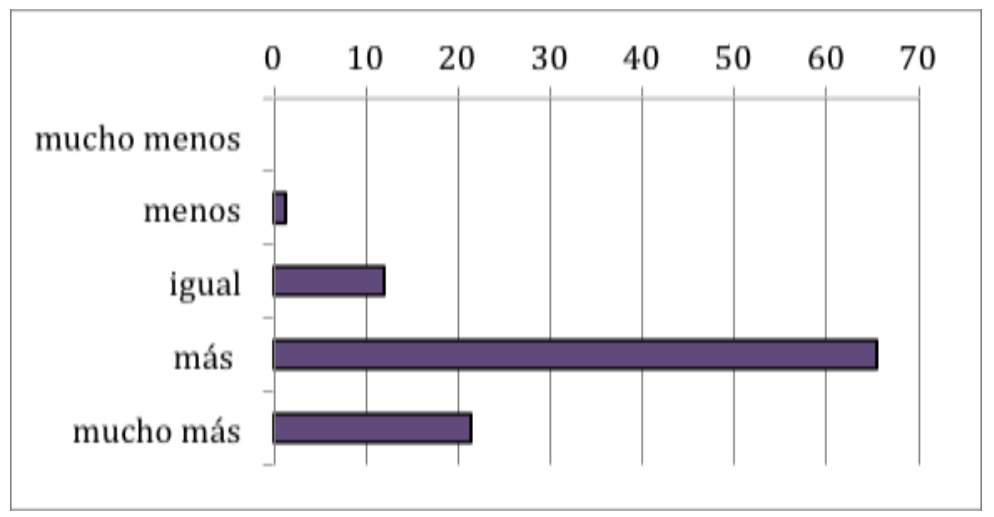

Figura 7. Valoración de los estudiantes del grado en que la asignatura les ha ayudado a aprender significativamente, en comparación con asignaturas de planteamiento tradicional

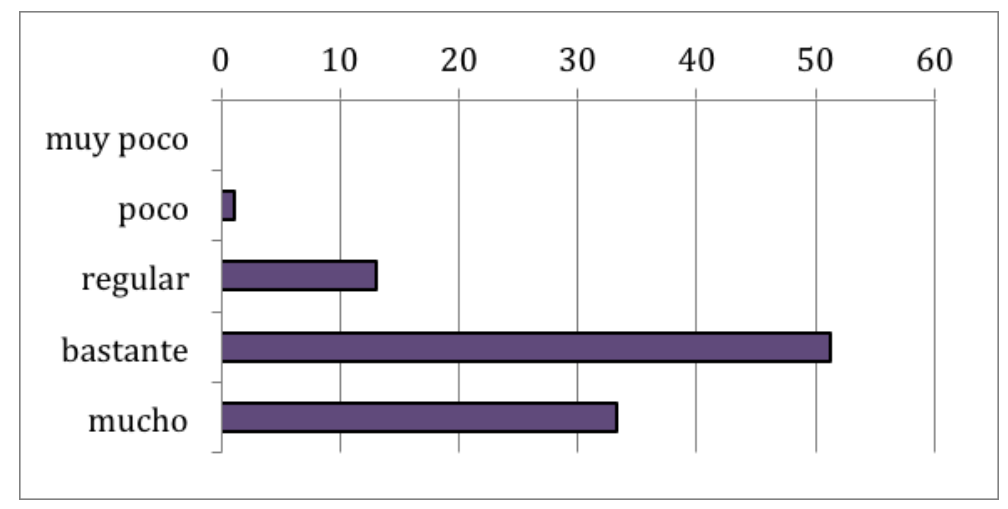

Figura 8. Satisfacción global de los estudiantes con la experiencia

En las respuestas a las preguntas abiertas, los estudiantes destacan como aspectos del planteamiento metodológico y didáctico de la asignatura que más les han ayudado a aprender las herramientas de aprendizaje puestas a su disposición, entre las que se incluye el "cuaderno de bitácora" (un $80 \%$ de los estudiantes hacen referencia en sus respuestas a una o varias de estas herramientas); la organización y distribución de las tareas y la entrega de las evidencias (un 50\% de los estudiantes remiten a este aspecto en sus respuestas); el trabajo colaborativo en grupo (un $45 \%$ de los estudiantes lo mencionan); y la retroalimentación continuada del profesor (un $32 \%$ de los estudiantes lo mencionan). Al mismo tiempo, señalan como aspecto fundamental a mejorar el ajuste de la carga y de las exigencias del trabajo de la asignatura (un $62 \%$ de los estudiantes hacen referencia en sus respuestas a esta cuestión). En cuanto a los resultados académicos (Tabla 2), destaca el alto porcentaje de estudiantes que superó la asignatura (el 85\%), así como el porcentaje de estudiantes con calificaciones iguales o superiores al notable (algo más del 70\%). 
Tabla 2. Calificaciones finales de los estudiantes en la asignatura

\begin{tabular}{lcc} 
Calificaciones & \multicolumn{2}{c}{ Estudiantes } \\
\hline Sobresaliente & 50 & \% \\
Notable & 41 & 39,4 \\
Aprobado & 17 & 32,3 \\
Suspenso & 4 & 13,4 \\
No presentado & 15 & 3,1 \\
TOTAL & 127 & 11,8 \\
\hline
\end{tabular}

En cuanto al profesorado, valoró positivamente la consecución de los objetivos del proyecto. Hubo coincidencia entre los profesores en que las principales aportaciones de la innovación para los estudiantes fueron una mayor toma de conciencia de sus procesos de trabajo; la adquisición de ciertas habilidades de planificación, supervisión y valoración de su proceso de aprendizaje; y una mayor implicación en las actividades. Al mismo tiempo, los profesores coincidieron en identificar algunos puntos débiles y dificultades en la experiencia. Así, señalaron que la complejidad de las evidencias solicitadas, y la carga de trabajo que comportaban para los estudiantes, podían provocar, en algunos casos, un abordaje formal y mecánico de las mismas, que no permitía aprovechar sus potencialidades. Ésta se consideró, además, la razón más importante de la eventual insatisfacción de los estudiantes con la experiencia. Igualmente, remarcaron la carga de trabajo que había supuesto para ellos mismos el seguimiento y la tutorización del trabajo de los estudiantes exigidos por la experiencia.

\section{Discusión y conclusiones}

Como se recordará, el objetivo de la experiencia de innovación realizada era el diseño y desarrollo de un conjunto de ayudas, vertebradas alrededor del "cuaderno de bitácora", dirigidas a apoyar el proceso de regulación individual y grupal de los estudiantes. A este respecto, los resultados presentados confirman las dificultades que encuentran los estudiantes para regular adecuadamente, de forma espontánea y sin ayudas específicas, sus procesos colaborativos. Así lo demuestra el hecho de que menos de la mitad de los estudiantes valoren positivamente sus procesos de trabajo individual y en pequeño grupo en el primer bloque de la asignatura. En este sentido, nuestro trabajo confirma los resultados obtenidos por diversos trabajos previos en el ámbito del CSCL que constatan la complejidad y dificultad que supone 
para los estudiantes coordinar y regular su trabajo en grupo (Engel y Onrubia, 2010; Manlove, Lazonder y de Jong, 2009; Mauri, Colomina, Clarà y Ginesta, 2011; Meier, Spada y Rummel, 2007; Schoor y Bannert, 2012). Ello también es coherente con la imagen multidimensional de los procesos de regulación que los trabajos en este ámbito han ido dibujando en los últimos años. Unos procesos que incluyen aspectos tanto de carácter cognitivo como social y afectivo o motivacional, y que combinan de manera compleja procesos de autorregulación, de regulación del otro y de regulación compartida (Chan, 2012; Järvelä y Hadwin, 2013; Saab, 2012; Schoor y Bannert, 2012).

Frente a estas dificultades, la intervención realizada obtiene resultados positivos tanto desde el punto de vista de la satisfacción de los estudiantes y del profesorado como desde el punto de vista de la mejora que los propios estudiantes perciben en sus procesos de organización y funcionamiento individuales y grupales. En este sentido, consideramos especialmente significativa la mejora que manifiestan los estudiantes en sus procesos de regulación individual y en grupo en el segundo bloque de la asignatura y la consolidación de esa mejora en el tercero. Estos resultados confirman la necesidad de apoyar y orientar las actuaciones de los estudiantes para facilitar que desarrollen interacciones óptimas desde el punto de vista de la construcción colaborativa del conocimiento en entornos CSCL. Son, en general, propuestas que reivindican la importancia del profesor en un doble papel. Por una parte, el papel del profesor como diseñador de aproximaciones instruccionales que pauten y estructuren explícitamente los procesos colaborativos de los estudiantes como, por ejemplo, mediante la elaboración de scripts de CSCL (Carmien, Kollar, Fischer y Fischer, 2007; Dillenbourg y Hong, 2008; Kirschner, Sweller y Clark, 2006). Por otra parte, el papel del profesor en el seguimiento escrupuloso de los procesos colaborativos efectivamente desarrollados por los grupos. Un seguimiento orientado a regular y andamiar la interacción entre los estudiantes para guiarlos hacia un aprendizaje y una colaboración efectivas, ofreciéndoles ayudas relacionadas tanto con los contenidos de aprendizaje como con la gestión de su colaboración, así como a adaptar, a partir de la información obtenida, el diseño previamente planificado (Fischer y Dillenbourg, 2006; Mauri, Colomina, Clarà y Ginesta, 2011; Onrubia y Engel, 2012).

En el marco de estos resultados positivos, la principal limitación de la intervención realizada que muestran los resultados obtenidos es el volumen y la carga de trabajo que supone el "cuaderno de bitácora" para los estudiantes, y también para los profesores. Así lo señalan tanto las respuestas abiertas al cuestionario de valoración de la asignatura por parte de los 
estudiantes como los autoinformes del profesorado. A este respecto y al margen de otras consideraciones, entendemos que nuestro intento de enseñar las competencias de regulación del aprendizaje individual y en grupo de manera simultánea a las tareas específicas de la asignatura ha podido dar lugar en algunos momentos a un fenómeno de sobrecarga cognitiva (Kirschner, 2002). Esta sobrecarga cognitiva se reflejaría en el hecho de que algunos estudiantes abordasen en algunos casos la elaboración de las evidencias de manera superficial, sin involucrarse en los procesos de toma de conciencia y reflexión sobre la colaboración que pretendíamos promover. Ello, junto con las apreciaciones del profesorado resaltando que el seguimiento y la tutorización de los grupos les habían supuesto un esfuerzo considerable de dedicación, apunta a la necesidad de introducir algunas modificaciones en la experiencia que ajusten la complejidad y el volumen de las evidencias solicitadas.

Los resultados obtenidos permiten también orientar algunas perspectivas de futuro y vías prioritarias de mejora de la intervención propuesta. La primera de ellas tiene que ver con conseguir un mejor ajuste de la carga de trabajo y la exigencia a los estudiantes a lo largo del proceso, sin que ello afecte a la eficacia de la intervención. Mejorar la experiencia desde este punto de vista parece crucial para asegurar que los beneficios de la innovación realizada puedan extenderse y mantenerse de forma sostenible. Dos líneas adicionales de trabajo futuro remiten a atender de manera más equilibrada tanto a los componentes cognitivos como a los componentes motivacionales y afectivos implicados en la regulación del trabajo colaborativo, así como flexibilizar los apoyos ofrecidos para que se adapten mejor a grupos y estudiantes con experiencia y habilidades diversas de trabajo en equipo. Todo ello dirigido a tratar de apoyar de maneras cada vez más ajustadas la mejora de las competencias de los estudiantes para regular su trabajo y su aprendizaje en entornos CSCL.

\section{Referencias}

Álvarez-Valdivia, I. y López-Benavides, D. (2010). Regulación del comportamiento durante la construcción conjunta de conocimientos en tareas cooperativas en entornos de aprendizaje virtuales asincrónicos y escritos. Cultura y Educación, 22(4), 419-438. doi: $10.1174 / 113564010793351830$

Bereiter, C. (1994). Implications of postmodernism for science, or, science as progressive discourse. Educational Psychologist, 29 (1), 3-12. 
doi: http://dx.doi.org/10.1207/s15326985ep2901_1

Boeckaerts, M. (2002). Bringing about change in the classroom: Strenghts and weaknesses of the self regulated learning approach. Learning and Instruction, 12, 589-604. http://dx.doi.org/10.1016/S0959-4752(02)00010-5

Carmien, S., Kollar, I., Fischer, G., \& Fischer, F. (2007). The interplay of internal and external scripts. In F. Fischer, I Kollar, H. Mandl \& J. M. Haake, Scripting computersupported collaborative learning (pp. 303-326). New York: Springer.

Chan, C.K.K. (2012) Co-regulation of learning in computer-supported collaborative learning environments: A discussion. Metacognition and Learning, 7 (1), 63-73. doi: http://dx.doi.org/10.1007/s11409-012-9086-z

De la Fuente, J. y Justicia, F. (2007). El modelo DIDEPRO de regulación de la enseñanza y del aprendizaje: avances recientes. Electronic Journal of Research in Educational Psychology, 5(3), 535-564.

De la Fuente, J., Justicia, F., Sander, P., \& Cardelle-Elawar, M. (2014). Personal SelfRegulation and Regulatory Teaching to predict Performance and Academic Confidence: new evidence for the DEDEPRO Model. Electronic Journal of Research in Educational Psychology, 12(3), 597-620. http://dx.doi.org/10.14204/ejrep.34.14031

Dillenbourg, P., Baker, M., Blaye, A. y O'Malley, C. (1996). The evolution of research on collaborative learning. En E. Spada y P. Reiman (Eds), Learning in Humans and Machine: Towards an interdisciplinary learning science (pp. 189-211). Oxford: Elsevier.

Dillenbourg, P. y Hong, F. (2008). The mechanics of CSCL macro scripts. International Journal of Computer-Supported Collaborative Learning, 3(1), 5-23. doi: http://dx.doi.org/10.1007/s11412-007-9033-1

Dillenbourg, P., Järvelä, S. y Fischer, F. (2009). The Evolution of Research on ComputerSupported Collaborative Learning. From Design to Orchestration. In N. Balacheff, S. Ludvigsen, T. De Jong, A. Lazonder y S. Barnes (Eds.), Technology-Enhanced Learning. Principles and Products (pp. 3-19). Dordrecht: Springer Netherlands.

Dillenbourg, P., Nussbaum, M., Dimitriadis, Y. y Roschelle, J. (en prensa). Design for Classroom Orchestration. Computers \& Education. doi: http://dx.doi.org/10.1016/j.compedu.2012.10.026

Engel, A. y Onrubia, J. (2010). Patrones de organización grupal y fases de construcción del conocimiento en entornos virtuales de aprendizaje colaborativo. Infancia y Aprendizaje, 33(4), 515-528. doi: http://dx.doi.org/10.1174/021037010793139608

Fischer, F. y Dillenbourg, P. (2006, April). Challenges of orchestrating computer-supported 
collaborative learning. Paper presented at the 87th. Annual Meeting of the American Educational Research Association (AERA), San Francisco, USA.

Garrison, D, R. y Anderson, T. (2003). E-learning in the 21st century. A framework for research and practice. London: RoutledgeFalmer [El e-learning en el siglo XXI: investigación y práctica. Barcelona: Octaedro].

Janssen, J., Erkens G., Kirschner P., y Kanselaar, G. (2012). Task-related and social regulation during online collaborative learning. Metacognition and Learning, 7(1), 25-43. doi: $10.1007 / \mathrm{s} 11409-010-9061-5$

Järvelä, S. y Hadwin, A. H. (2013). New Frontiers: Regulating Learning in CSCL. Educational Psychologist, 48(1), 25-39. doi: 10.1080/00461520.2012.748006

Johnson, D. W. y Johnson, R. T. (1974). Instructional goal structure: Cooperative competitive or individualistic. Review of Education Research, 44, 213-240.

Kirschner, P. A. (Ed.) (2002). Cognitive load theory [Special Issue]. Learning and Instruction, 12. doi: http://dx.doi.org/10.1016/S0959-4752(01)00014-7

Kirschner, P.A., Sweller, J. y Clark, R. E. (2006). Why Minimal Guidance During Instruction Does Not Work: An Analysis of the Failure of Constructivist, Discovery, ProblemBased, Experiential, and Inquiry-Based Teaching. Educational Psychologist, 41(2), 7586. doi: http://dx.doi.org/10.1207/s15326985ep4102_1

Lipponen, L. y Lallimo, J. (2004). Assessing applications for collaboration: from collaboratively usable applications to collaborative technology. British Journal of Educational Technology, 35(4), 433-442. doi: http://dx.doi.org/10.1111/j.0007-1013.2004.00402.x

Manlove, S., Lazonder, A.W. y De Jong, T. (2009). Trends and issues of regulative support use during inquiry learning: Patterns from three studies. Computers in Human Behavior, 25 (4), 795-803. doi: http://dx.doi.org/10.1016/j.chb.2008.07.010

Martínez, A., Dimitriadis, Y. y De La Fuente, P. (2003). Interaction analysis for formative evaluation in CSCL. In M. Llamas, M. J. Fernández, y L. E. Anido (Ed.), Computers and Education: Toward a lifelong learning society (pp. 227-238). Dordrecht : Kluwer Academic.

Mauri, T., Colomina, R., Clarà, M. y Ginesta, A. (2011). Ayudas al aprendizaje en tareas de escritura colaborativa con Moodle. Electronic Journal of Research in Educational Psychology, 9(3), 1103-1128.

Meier, A., Spada, H. y Rummel, N. (2007). A rating scheme for assessing the quality of computer-supported collaboration processes. International Journal of Computer-Supported Collaborative Learning, 2 (1), 63-86. doi: http://dx.doi.org/10.1007/s11412-006-9005-x 
Mercer, N. (2001). Palabras y mentes. Cómo usamos el lenguaje para pensar juntos. Barcelona: Paidós.

Onrubia, J. y Engel, A. (2012). The role of teacher assistance on the effects of a macro-script in collaborative writing tasks. International Journal of Computer-Supported Collaborative Learning, 7 (1), 161-186. doi: http://dx.doi.org/10.1007/s11412-011-9125-9

Pifarré, M. y Cobos, R. (2010). Promoting metacognitive skills through peer scaffolding in a CSCL environment. International Journal of Computer-Supported Collaborative Learning, 5(2), 237-253. doi: http://dx.doi.org/10.1007/s11412-010-9084-6

Pintrich, P. (2000). The role of goal orientation in self-regulated learning. En M. Boekaerts, P. Pintrich \& M. Zeidner (Eds.), Handbook of Self-Regulation (pp. 451-502). California: Academic Press. doi: 10.1016/B978-012109890-2/50043-3

Resta, P. y Laferrière, T. (2007). Technology in Support of Collaborative Learning. Educational Psychology Review, 19(1), 65-83. doi: http://dx.doi.org/10.1007/s10648-0079042-7

Saab, N. (2012). Team regulation, regulation of social activities or co-regulation: Different labels for effective regulation of learning in CSCL. Metacognition and Learning, 7 (1), 1-6. doi: http://dx.doi.org/10.1007/s11409-012-9086-z

Saab, N., Joolingen, W. y Hout-Wolters, B. (2012). Support of the collaborative inquiry learning process: Influence of support on task and team regulation. Metacognition and Learning, 7(1), 7-23. DOI 10.1007/s11409-011-9068-6

Scardamalia, M. y Bereiter, C. (2003). Knowledge building. En J. W. Guthrie (Ed.), Encyclopedia of education (2 ${ }^{\mathrm{a}}$ ed., pp. 1370-1373). Nueva York: Macmillan Reference, USA.

Schoor, C. y Bannert, M. (2012). Exploring regulatory processes during a computer-supported collaborative learning task using process mining. Computers in Human Behavior, 28 (4), 1321-1331. doi: http://dx.doi.org/10.1016/j.chb.2012.02.016

Slavin, R. E. (1996). Research for the future: Research on cooperative learning and achievement: What we know, what we need to know. Contemporary Educational Psychology, 21, 43-69. doi: http://dx.doi.org/10.1006/ceps.1996.0004

Stahl, G., Koschmann, T. y Suthers, D. (2006). Computer-supported collaborative learning: An historical perspective. En R. K. Sawyer (Ed.), Cambridge handbook of the learning sciences. Cambridge, UK: Cambridge University Press.

Suthers, D. (2006). Technology affordances for intersubjective meaning making: A research agenda for CSCL. Computer-Supported Collaborative Learning, 1, 315-337. doi: http://dx.doi.org/10.1007/s11412-006-9660-y 
Tabak, I. (2004). Synergy: a complement to emerging patterns of distributed scaffolding. Journal of the Learning Sciences, 13 (3), 305-335. doi: http://dx.doi.org/10.1207/s15327809j1s1303_3

Volet, S., Summers, M., \& Thurman, J. (2009). High-level co-regulation in collaborative learning: How does it emerge and how is it sustained? Learning and Instruction, 19(2), 128-143. http://dx.doi.org/10.1016/j.learninstruc.2008.03.001 